Volume 28 (2020) 61-74

DOI: $10.24330 /$ ieja. 768135

\title{
ON THE DOT PRODUCT GRAPH OF A COMMUTATIVE RING
} II

\author{
Mohammad Abdulla and Ayman Badawi \\ Received: 3 August 2019; Revised: 20 December 2019; Accepted: 5 January 2020 \\ Communicated by Sarah Glaz
}

\begin{abstract}
In 2015, the second-named author introduced the dot product graph associated to a commutative ring $A$. Let $A$ be a commutative ring with nonzero identity, $1 \leq n<\infty$ be an integer, and $R=A \times A \times \cdots \times A$ ( $n$ times). We recall that the total dot product graph of $R$ is the (undirected) graph $T D(R)$ with vertices $R^{*}=R \backslash\{(0,0, \ldots, 0)\}$, and two distinct vertices $x$ and $y$ are adjacent if and only if $x \cdot y=0 \in A$ (where $x \cdot y$ denotes the normal dot product of $x$ and $y$ ). Let $Z(R)$ denote the set of all zero-divisors of $R$. Then the zero-divisor dot product graph of $R$ is the induced subgraph $Z D(R)$ of $T D(R)$ with vertices $Z(R)^{*}=Z(R) \backslash\{(0,0, \ldots, 0)\}$. Let $U(R)$ denote the set of all units of $R$. Then the unit dot product graph of $R$ is the induced subgraph $U D(R)$ of $T D(R)$ with vertices $U(R)$. In this paper, we study the structure of $T D(R), U D(R)$, and $Z D(R)$ when $A=Z_{n}$ or $A=G F\left(p^{n}\right)$, the finite field with $p^{n}$ elements, where $n \geq 2$ and $p$ is a prime positive integer.
\end{abstract}

Mathematics Subject Classification (2020): 13A15, 13B99, 05C99

Keywords: Dot product graph, annihilator graph, total graph, zero-divisor graph

\section{Introduction}

Let $R$ be a commutative ring with $1 \neq 0$. Then $Z(R)$ denote the set of zerodivisors of $R$ and the group of units of $R$ will be denoted by $U(R)$. As usual, $Z_{n}$ denotes the ring of integers modulo $n$. The nonzero elements of $S \subseteq R$ will be denoted by $S^{*}$. Over the past several years, there has been considerable attention in the literature to associating graphs with commutative rings (and other algebraic structures) and studying the interplay between ring-theoretic and graph-theoretic properties (for example, see [1]-[20] and [22]-[26]). In particular, as in [9], the zero-divisor graph of $R$ is the (simple) graph $\Gamma(R)$ with vertices $Z(R) \backslash\{0\}$, and distinct vertices $x$ and $y$ are adjacent if and only if $x y=0$. This concept is due to Beck [16], who let all the elements of $R$ be vertices and was mainly interested in 
coloring. The zero-divisor graph of a ring $R$ has been studied extensively by many authors.

In 2015, Badawi [15] introduced the dot product graph associated to a commutative ring $A$. Let $A$ be a commutative ring with nonzero identity, $1 \leq n<\infty$ be an integer, and $R=A \times A \times \cdots \times A$ ( $n$ times). We recall from [1] that the total dot product graph of $R$ is the (undirected) graph $T D(R)$ with vertices $R^{*}=R \backslash\{(0,0, \ldots, 0)\}$, and two distinct vertices $x$ and $y$ are adjacent if and only if $x \cdot y=0 \in A$ (where $x \cdot y$ denotes the normal dot product of $x$ and $y)$. Let $Z(R)$ denote the set of all zero-divisors of $R$. Then the zero-divisor dot product graph of $R$ is the induced subgraph $Z D(R)$ of $T D(R)$ with vertices $Z(R)^{*}=Z(R) \backslash\{(0,0, \ldots, 0)\}$. Let $U(R)$ denote the set of all units of $R$. Then the unit dot product graph of $R$ is the induced subgraph $U D(R)$ of $T D(R)$ with vertices $U(R)$. Let $p \geq 2$ be a prime integer, $n \geq 1, A=G F\left(p^{n}\right)$ be the finite field with $p^{n}$ elements, and $R=A \times A$. In Section 2 of this paper, we study the structure of $Z D(R), U D(R)$, and $T D(R)$. Let $n \geq 2$, $A=Z_{n}$, and $R=A \times A$. In Section 3 of this paper, we study the structure of $U D(R)$. In Section 4, we study some induced subgraphs of $Z D\left(Z_{n} \times Z_{n}\right)$, where $n \geq 2$. In Section 5 , we introduce the equivalence unit dot product of $R, E U D(R)$ and we show that $U D(R)$, can be recovered from $E U D(R)$.

Let $G$ be a graph. Two vertices $v_{1}, v_{2}$ of $G$ are said to be adjacent in $G$ if $v_{1}, v_{2}$ are connected by an edge (line segment) of $G$ and we write $v_{1}-v_{2}$. A finite sequence of edges from a vertex $v_{1}$ of $G$ to a vertex $v_{2}$ of $G$ is called a path of $G$ and we write $v_{1}-a_{1}-a_{2}-\cdots-a_{k}-v_{2}$, where $k<\infty$ and the $a_{i}, 1 \leq i \leq k$, are some distinct vertices of $G$. Hence it is clear that every edge of $G$ is a path of $G$, but not every path of $G$ is an edge of $G$. We say that $G$ is connected if there is a path between any two distinct vertices of $G$. At the other extreme, we say that $G$ is totally disconnected if no two vertices of $G$ are adjacent. We denote the complete graph on $n$ vertices by $K_{n}$, recall that a graph $G$ is called complete if every two vertices of $G$ are adjacent and the complete bipartite graph on $m$ and $n$ vertices by $K_{m, n}$ (recall that $K_{m, n}$ is the graph with two sets of vertices, say $V_{1}, V_{2}$, such that $\left|V_{1}\right|=n,\left|V_{2}\right|=m, V_{1} \cap V_{2}=\emptyset$, every two vertices in $V_{1}$ are not adjacent, every two vertices in $V_{2}$ are not adjacent, and every vertex in $V_{1}$ is adjacent to every vertex in $V_{2}$ ). We will sometimes call a $K_{1, n}$ a star graph. We say that two (induced) subgraphs $G_{1}$ and $G_{2}$ of $G$ are disjoint if $G_{1}$ and $G_{2}$ have no common vertices and no vertex of $G_{1}$ (resp., $G_{2}$ ) is adjacent (in $G$ ) to any vertex not in $G_{1}$ (resp., $G_{2}$ ). Assume that a graph $G=G_{1} \cup G_{2} \cup \cdots \cup G_{n}$, where each vertex of $G_{i}$ 
is not connected to a vertex of $G_{j}$ for every $1 \leq i, j \leq n$ with $i \neq j$. Then we say that $G$ is the disjoint union of $G_{1}, \ldots, G_{n}$.

\section{The structure of $U D(R=A \times A)$ when $A$ is a field}

Let $p$ be a positive prime number, $n \geq 2$. Then $A=G F\left(p^{n}\right)$ denotes the finite field with $p^{n}$ elements. Let $R=A \times A$. Then $T D(R)$ is not connected by [15, Theorem 2.1]. The first two results give a complete description of the structure of $U D(R)$ and $T D(R)$.

Theorem 2.1. Let $n \geq 1, m=2^{n}-1$ and $R=G F\left(2^{n}\right) \times G F\left(2^{n}\right)$. Then

(1) $Z D(R)=\Gamma(R)=K_{m, m}$.

(2) $U D(R)$ is the disjoint union of one $K_{m}$ and $\left(2^{(n-1)}-1\right) K_{m, m}$ 's.

(3) $T D(R)$ is the disjoint union of one $K_{m}$ and $2^{(n-1)} K_{m, m}$ 's.

Proof. (1) The result is clear by [15, Theorem 2.1], [9, Theorem 2.1], and [10, Theorem 2.2].

(2) Let $A=G F\left(2^{n}\right)$. Then $R=A \times A$. Let $v_{1}, v_{2} \in U(R)$. Since $R$ is a vector space over $A, v_{1}=u(1, a) \in R$ and $v_{2}=v(1, b) \in R$ for some $u, v, a, b \in A^{*}$. Hence $v_{1}$ is adjacent to $v_{2}$ if and only if $v_{1} \cdot v_{2}=u v+u v a b=0$ in $A$ if and only if $b=-a^{-1}=a^{-1}$ in $A$. Thus for each $a \in U(A)=A^{*}$, let $X_{a}=\left\{u(1, a) \mid u \in A^{*}\right\}$ and $Y_{a}=\left\{u\left(1, a^{-1}\right) \mid u \in A^{*}\right\}$. It is clear that $\left|X_{a}\right|=\left|Y_{a}\right|=2^{n}-1$. Let $a=1$. Since $\operatorname{char}(A)=\operatorname{char}(R)=2, X_{a}=Y_{a}$ and the dot product of every two distinct vertices in $X_{a}$ is zero. Hence every two distinct vertices in $X_{a}$ are adjacent. Thus the vertices in $X_{a}$ form the graph $K_{m}$ that is a complete subgraph of $T D(R)$. Let $a \in U(A)$ such that $a \neq 1$. Since $a^{2} \neq 1$ for each $a \in U(A) \backslash\{1\}$, we have $X_{a} \cap Y_{a}=\emptyset$, every two distinct vertices in $X_{a}$ are not adjacent, and every two distinct vertices in $Y_{a}$ are not adjacent. Since $\operatorname{char}(A)=\operatorname{char}(R)=2$, it is clear that every vertex in $X_{a}$ is adjacent to every vertex in $Y_{a}$. Thus the vertices in $X_{a} \cup Y_{a}$ form the graph $K_{m, m}$ that is a complete bi-partite subgraph of $T D(R)$. By construction, there are exactly $\left(2^{n}-2\right) / 2=2^{n-1}-1$ disjoint complete bi-partite $K_{m, m}$ subgraphs of $T D(R)$. Hence $U D(R)$ is the disjoint union of one complete subgraph $K_{m}$ and $\left(2^{n-1}-1\right)$ complete bi-partite $K_{m, m}$ subgraphs.

(3) The claim follows from (1) and (2).

Theorem 2.2. Let $p \geq 3$ be a positive prime integer, $n \geq 1, m=p^{n}-1$, and let $R=G F\left(p^{n}\right) \times G F\left(p^{n}\right)$. Then

(1) $Z D(R)=\Gamma(R)=K_{m, m}$.

(2) If $4 \nmid m$, then $U D(R)$ is the disjoint union of $m / 2 K_{m, m}$ 's. 
(3) If $4 \mid m$, then $U D(R)$ is the disjoint union of two $K_{m}$ 's and $(m-2) / 2$ $K_{m, m}$ 's.

(4) If $4 \nmid m$, then $T D(R)$ is the disjoint union of $(m+2) / 2 K_{m, m}$ 's.

(5) If $4 \mid m$, then $T D(R)$ is the disjoint union of two $K_{m}$ 's and $m / 2 K_{m, m}$ 's.

Proof. (1) The result is clear by [15, Theorem 2.1], [9, Theorem 2.1], and [10, Theorem 2.2].

(2) Let $A=G F\left(p^{n}\right)$. Then $R=A \times A$. Let $v_{1}, v_{2} \in U(R)$. Since $R$ is a vector space over $A, v_{1}=u(1, a) \in R$ and $v_{2}=v(1, b) \in R$ for some $u, v, a, b \in A^{*}$. Hence $v_{1}$ is adjacent to $v_{2}$ if and only if $v_{1} \cdot v_{2}=u v+u v a b=0$ in $A$ if and only if $b=-a^{-1}$ in $A$. Since $R$ is a vector space over $A$, for each $a \in U(A)=A^{*}$, let $X_{a}=\left\{u(1, a) \mid u \in A^{*}\right\}$ and $Y_{a}=\left\{u\left(1,-a^{-1}\right) \mid u \in A^{*}\right\}$. It is clear that $\left|X_{a}\right|=\left|Y_{a}\right|=m=p^{n}-1$. Since $4 \nmid m, U(A)=A^{*}$ has no elements of order 4 . Thus $a^{2} \neq-1$ for each $a \in U(A)$. Hence $X_{a} \cap Y_{a}=\emptyset$; so every two distinct vertices in $X_{a}$ are not adjacent, and every two distinct vertices in $Y_{a}$ are not adjacent. By construction of $X_{a}$ and $Y_{a}$, it is clear that every vertex in $X_{a}$ is adjacent to every vertex in $Y_{a}$. Thus the vertices in $X_{a} \cup Y_{a}$ form the graph $K_{m, m}$ that is a complete bi-partite subgraph of $T D(R)$. By construction, there are exactly $m / 2$ disjoint complete bi-partite $K_{m, m}$ subgraphs of $T D(R)$. Hence $U D(R)$ is the disjoint union of $m / 2 K_{m, m}$ 's.

(3) Note that $|U(A)|=m$. Since $U(A)=A^{*}$ is cyclic and $4 \mid m, U(A)$ has exactly one subgroup of order 4 . Thus $U(A)$ has exactly two elements of order 4 , say $b, c$. Since $a \in U(A)$ is of order 4 if and only if $a^{2}=-1$, it is clear that $x^{2}=-1$ for some $x \in U(A)$ if and only if $x=b, c$. Let $X_{b}=\{u(1, b) \mid u \in U(A)\}$ and let $X_{c}=\{u(1, c) \mid u \in U(A)\}$. It is clear that $\left|X_{b}\right|=\left|X_{c}\right|=m$. Let $H=\{b, c\}$. Then the dot product of every two distinct vertices in $X_{h}$ is zero for each $h \in H$. Thus every two distinct vertices in $X_{h}$ are adjacent for every $h \in H$. Thus for each $h \in H$, the vertices in $X_{h}$ form the graph $K_{m}$ that is a complete subgraph of $T D(R)$. Let $a \in U(A) \backslash H, X_{a}=\left\{u(1, a) \mid u \in A^{*}\right\}$, and $Y_{a}=\left\{u\left(1,-a^{-1}\right) \mid u \in A^{*}\right\}$. It is clear that $\left|X_{a}\right|=|Y a|=m$. Since $a \notin H$, we have $X_{a} \cap Y_{a}=\emptyset$, every two distinct vertices in $X_{a}$ are not adjacent, and every two distinct vertices in $Y_{a}$ are not adjacent. By construction, it is clear that every vertex in $X_{a}$ is adjacent to every vertex in $Y_{a}$. Thus the vertices in $X_{a} \cup Y_{a}$ form the graph $K_{m, m}$ that is a complete bi-partite subgraph of $T D(R)$. By construction, there are $(m-2) / 2$ disjoint $K_{m, m}$ subgraphs. Hence $U D(R)$ is the disjoint union of two $K_{m}$ 's and $(m-2) / 2 \quad K_{m, m}$ 's.

(4) The claim follows from (1) and (2). 
(5) The claim follows from (1) and (3).

In view of Theorem 2.2 , we have the following corollary.

Corollary 2.3. Let $p \geq 3$ be a prime positive integer, and let $R=Z_{p} \times Z_{p}$. Then

(1) $Z D(R)=\Gamma(R)=K_{p-1, p-1}$.

(2) If $4 \nmid p-1$, then $U D(R)$ is the disjoint union of $(p-1) / 2 K_{p-1, p-1}$.

(3) If $4 \mid p-1$, then $U D(R)$ is the disjoint union of two $K_{p-1}$ 's and $(p-3) / 2$ $K_{p-1, p-1}$ 's.

(4) If $4 \nmid p-1$, then $T D(R)$ is the disjoint union of $(p+1) / 2 K_{p-1, p-1}$ 's.

(5) If $4 \mid p-1$, then $T D(R)$ is the disjoint union of two $K_{p-1}$ 's and $(p-1) / 2$ $K_{p-1, p-1}$ 's.

Example 2.4. Let $A=\frac{Z_{2}[X]}{\left(X^{2}+X+1\right)}$. Then $A$ is a finite field with 4 elements. Let $v=X+\left(X^{2}+X+1\right) \in A$. Since $\left(A^{*},.\right)$ is a cyclic group and $A^{*}=<v>$, we have $A=\left\{0, v, v^{2}, v^{3}=1+\left(X^{2}+X+1\right)\right\}$. Let $R=A \times A$. Then the $U D(R)$ is the disjoint union of one $K_{3}$ and one $K_{3,3}$ by Theorem 2.1(2). The following is the graph of $U D(R)$.
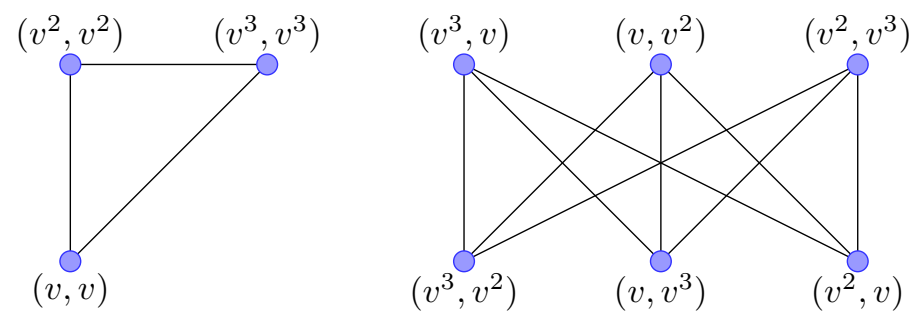

Example 2.5. Let $A=Z_{5}$ and $R=A \times A$. Then $U D(R)$ is the disjoint union of two $K_{4}$ and one $K_{4,4}$ by Corollary 2.3(3). The following is the graph of $U D(R)$.
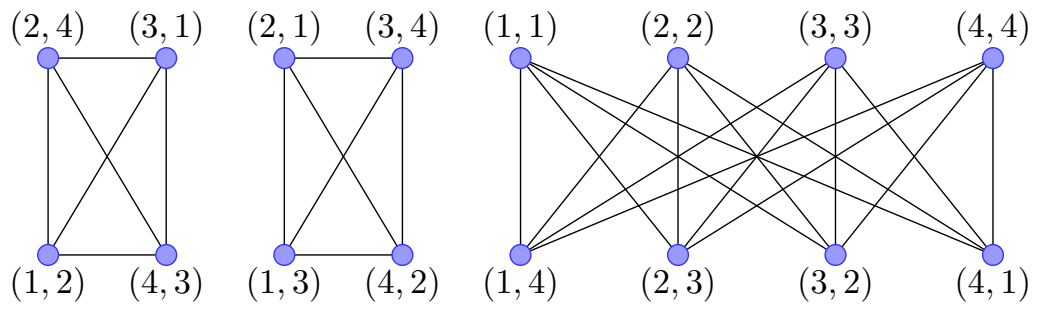

3. Unit dot product graph of $R=Z_{n} \times Z_{n}$

Let $n>1$ and write $n=p_{1}^{k_{1}} \cdots p_{m}^{k_{m}}$, where the $p_{i}$ 's are distinct prime positive integers. Then $U\left(Z_{n}\right)=\{1 \leq a<n \mid$ a is an integer and $\operatorname{gcd}(a, n)=1\}$. It is 
known that $U\left(Z_{n}\right)$ is a group under multiplication modulo $n$ and $\left|U\left(Z_{n}\right)\right|=\phi(n)=$ $\left(p_{1}-1\right) p_{1}^{k_{1}-1}\left(p_{2}-1\right) p_{2}^{k_{2}-1} \cdots\left(p_{m}-1\right) p_{m}^{k_{m}-1}$.

The following lemma is needed.

Lemma 3.1. Let $n$ be a positive integer and write $n=p_{1}^{k_{1}} p_{2}^{k_{2}} \cdots p_{r}^{k_{r}}$, where the $p_{i}$ 's are distinct prime positive integers. Then

(1) If $4 \mid n$, then $a^{2} \not \equiv n-1 \quad(\bmod n)$ for each $a \in U\left(Z_{n}\right)$.

(2) If $4 \nmid n$, then $x^{2} \equiv n-1$ (mod $n$ ) has a solution in $U\left(Z_{n}\right)$ if and only if $4 \mid\left(p_{i}-1\right)$ for each odd prime factor $p_{i}$ of $n$. Furthermore, if $x^{2} \equiv$ $n-1(\bmod n)$ has a solution in $U\left(Z_{n}\right)$, then it has exactly $2^{r-1}$ distinct solutions in $U\left(Z_{n}\right)$ if $n$ is even and it has exactly $2^{r}$ distinct solutions in $U\left(Z_{n}\right)$ if $n$ is odd.

Proof. (1) Suppose that $4 \mid n$. Then $n \geq 4$. Since $4 \nmid(n-2), n-1 \not \equiv 1(\bmod 4)$ and thus $a^{2} \not \equiv n-1(\bmod \mathrm{n})$ for each $a \in U\left(Z_{n}\right)$ by [19, Theorem 5.1].

(2) Suppose that $4 \nmid n$. Then $a^{2} \equiv n-1(\bmod n)$ for some $a \in U\left(Z_{n}\right)$ if and only if $a^{2} \equiv n-1\left(\bmod p_{i}\right)$ for each odd prime factor $p_{i}$ of $n$ by [19, Theorem 5.1]. Thus $a^{2} \equiv n-1(\bmod n)$ for some $a \in U\left(Z_{n}\right)$ if and only if $\left(a \bmod p_{i}\right)^{2} \equiv p_{i}-1\left(\bmod p_{i}\right)$ for each odd prime factor $p_{i}$ of $n$. Since $U\left(Z_{p_{i}}\right)=Z_{p_{i}}^{*}=\left\{1, \ldots, p_{i}-1\right\}$ for each prime factor $p_{i}$ of $n$, we have $\left|U\left(Z_{p_{i}}\right)\right|=p_{i}-1$. For each $x \in U\left(Z_{p_{i}}\right), 1 \leq i \leq r$, let $|x|$ denotes the order of $x$ in $U\left(Z_{p_{i}}\right)$. Let $p_{i}, 1 \leq i \leq r$, be an odd prime factor of $n$. Since $\left|p_{i}-1\right|=2$ in $U\left(Z_{p_{i}}\right), b^{2}=p_{i}-1$ in $U\left(Z_{p_{i}}\right)$ for some $b \in U\left(Z_{p_{i}}\right)$ if and only if $|b|=4$ in $U\left(Z_{p_{i}}\right)$. Since $\left|U\left(Z_{p_{i}}\right)\right|=p_{i}-1$, we conclude that $b^{2}=p_{i}-1$ in $U\left(Z_{P_{i}}\right)$ for some $b \in U\left(Z_{p_{i}}\right)$ if and only if $4 \mid\left(p_{i}-1\right)$. Thus $x^{2} \equiv n-1(\bmod n)$ has a solution in $U\left(Z_{n}\right)$ if and only if $4 \mid\left(p_{i}-1\right)$ for each odd prime $p_{i}$ factor of $n$. Suppose that $x^{2} \equiv n-1(\bmod n)$ has a solution in $U\left(Z_{n}\right)$. We consider two cases: Case 1. Suppose that $n$ is an even integer. Then there are exactly $r-1$ distinct odd prime factors of $n$. Since $4 \nmid n, x^{2} \equiv n-1(\bmod n)$ has exactly $2^{r-1}$ distinct solutions in $U\left(Z_{n}\right)$ by [19, Theorem 5.2]. Case 2. Suppose that $n$ is an odd integer. Then there are exactly $r$ distinct odd prime factors of $n$. Thus $x^{2} \equiv n-1(\bmod n)$ has exactly $2^{r}$ distinct solutions in $U\left(Z_{n}\right)$ by [19, Theorem 5.2].

Let $A=Z_{n}$, where $n$ is not prime. Then $T D(A \times A)$ is connected by [15, Theorem 2.3 ]. In the following result, we show that $U D(A \times A)$ is disconnected, and we give a complete description of the structure of $U D(A \times A)$.

Theorem 3.2. Let $n \geq 3$ be an integer, $R=Z_{n} \times Z_{n}$ and $\phi(n)=m$. Write $n=p_{1}^{k_{1}} p_{2}^{k_{2}} \cdots p_{r}^{k_{r}}$, where the $p_{i}$ 's are distinct prime positive integers, $1 \leq i \leq r$. Then 
(1) If $4 \mid n$, then $U D(R)$ is the disjoint union of $m / 2 K_{m, m}$ 's.

(2) If $4 \nmid n$ and $4 \nmid\left(p_{i}-1\right)$ for at least one of the $p_{i}$ 's in the prime factorization of $n$, then $U D(R)$ is the disjoint union of $m / 2 K_{m, m}$ 's.

(3) If $4 \nmid n$ and $4 \mid\left(p_{i}-1\right)$ for all the odd $p_{i}$ 's in the prime factorization of $n$, then we consider two cases:

Case I. If $n$ is even, then $U D(R)$ is the disjoint union of $(m / 2)-2^{r-2}$ $K_{m, m}$ 's and $2^{r-1} K_{m}$ 's.

Case II. If $n$ is odd, then $U D(R)$ is the disjoint union of $(m / 2)-2^{r-1}$ $K_{m, m}$ 's and $2^{r} K_{m}$ 's.

Proof. Let $A=Z_{n}$. Then $R=A \times A$. Note that $U D(R)$ has exactly $m^{2}$ vertices. Let $v_{1}, v_{2} \in U(R)$. Since $R$ is a vector space over $A, v_{1}=u(1, a) \in R$ and $v_{2}=v(1, b) \in R$ for some $u, v, a, b \in U(A)$. Hence $v_{1}$ is adjacent to $v_{2}$ if and only if $v_{1} \cdot v_{2}=u v+u v a b=0$ in $A$ if and only if $b=-a^{-1}$ in $A$. Thus for each $a \in U(A)$, let $X_{a}=\{u(1, a) \mid u \in U(A)\}$ and $Y_{a}=\left\{u\left(1,-a^{-1}\right) \mid u \in U(A)\right\}$. It is clear that $\left|X_{a}\right|=\left|Y_{a}\right|=m$.

(1) Since $4 \mid n, a^{2} \not \equiv n-1 \quad(\bmod n)$ for each $a \in U\left(Z_{n}\right)$ by Lemma 3.1(1). Hence $X_{a} \cap Y_{a}=\emptyset$; so every two distinct vertices in $X_{a}$ are not adjacent, and every two distinct vertices in $Y_{a}$ are not adjacent. By construction of $X_{a}$ and $Y_{a}$, it is clear that every vertex in $X_{a}$ is adjacent to every vertex in $Y_{a}$. Thus the vertices in $X_{a} \cup Y_{a}$ form the graph $K_{m, m}$ that is a complete bi-partite subgraph of $T D(R)$. By construction, there are exactly $m / 2$ disjoint complete bi-partite $K_{m, m}$ subgraphs of $T D(R)$. Hence $U D(R)$ is the disjoint union of $m / 2 K_{m, m}$ 's.

(2) Write $n=p_{1}^{k_{1}} p_{2}^{k_{2}} \cdots p_{r}^{k_{r}}$, where the $p_{i}$ 's are distinct prime positive integers. Since $4 \nmid n$ and $4 \nmid\left(p_{i}-1\right)$ for at least one of the $p_{i}$ 's, $a^{2} \not \equiv n-1(\bmod n)$ for each $a \in U\left(Z_{n}\right)$ by Lemma 3.1. Thus by the same argument as in $(1), U D(R)$ is the disjoint union of $m / 2 K_{m, m}$ 's.

(3) Write $n=p_{1}^{k_{1}} p_{2}^{k_{2}} \cdots p_{r}^{k_{r}}$, where the $p_{i}$ 's are distinct prime positive integers. Suppose that $4 \nmid n$ and $4 \mid p_{i}-1$ for all the odd $p_{i}$ 's in the prime factorization of $n$. Let $B=\left\{b \in U\left(Z_{n}\right) \mid b^{2}=n-1\right.$ in $\left.U\left(Z_{n}\right)\right\}$ and $C=\left\{c \in U\left(Z_{n}\right) \mid c^{2} \neq n-1\right.$ in $\left.U\left(Z_{n}\right)\right\}$. We consider two cases: Case I. Suppose that $n$ is even. Then $|B|=2^{r-1}$ by Lemma 3.1(2) and hence $|C|=m-2^{r-1}$. For each $a \in B$, we have $X_{a}=Y_{a}$ and hence the dot product of every two distinct vertices in $X_{a}$ is zero. Thus the vertices in $X_{a}$ form the graph $K_{m}$ that is a complete subgraph of $T D(R)$. Hence $U D\left(Z_{n}\right)$ has exactly $2^{r-1}$ disjoint $K_{m}$ 's. For each $a \in C$, we have $X_{a} \cap Y_{a}=\emptyset$; so every two distinct vertices in $X_{a}$ are not adjacent, and every two distinct vertices in $Y_{a}$ are not adjacent. By construction, it is clear that every vertex in $X_{a}$ is adjacent to every 
vertex in $Y_{a}$. Thus the vertices in $X_{a} \cup Y_{a}$ form the graph $K_{m, m}$ that is a complete bi-partite subgraph of $T D(R)$. Thus $U D\left(Z_{n}\right)$ has exactly $\frac{m-2^{r-1}}{2}=\frac{m}{2}-2^{r-2}$ disjoint $K_{m, m}$ 's. Case II. Suppose that $n$ is odd. Then $|B|=2^{r}$ by Lemma 3.1(2) and hence $|C|=m-2^{r}$. For each $a \in B$, we have $X_{a}=Y_{a}$ and hence the dot product of every two distinct vertices in $X_{a}$ is zero. Thus the vertices in $X_{a}$ form the graph $K_{m}$ that is a complete subgraph of $T D(R)$. Hence $U D\left(Z_{n}\right)$ has exactly $2^{r}$ disjoint $K_{m}$ 's. For each $a \in C$, we have $X_{a} \cap Y_{a}=\emptyset$, every two distinct vertices in $X_{a}$ are not adjacent, and every two distinct vertices in $Y_{a}$ are not adjacent. By construction, it is clear that every vertex in $X_{a}$ is adjacent to every vertex in $Y_{a}$. Thus the vertices in $X_{a} \cup Y_{a}$ form the graph $K_{m, m}$ that is a complete bipartite subgraph of $T D(R)$. Thus $U D\left(Z_{n}\right)$ has exactly $\frac{m-2^{r}}{2}=\frac{m}{2}-2^{r-1}$ disjoint $K_{m, m}$ 's.

Recall that a graph $G$ is called completely disconnected if every two vertices of $G$ are not connected by an edge in $G$.

Theorem 3.3. Let $n \geq 4$ be an even integer, and let $R=Z_{n} \times Z_{n} \times \cdots \times Z_{n}$ (k times), where $k$ is an odd positive integer. Then $U D(R)$ is completely disconnected.

Proof. Let $x=\left(x_{1}, \ldots, x_{k}\right), y=\left(y_{1}, \ldots, y_{k}\right) \in U(R)$. Then $x_{i}, y_{i} \in U\left(Z_{n}\right)$ for every i, $1 \leq i \leq k$. Since $n$ is an even integer, $x_{i}$ and $y_{i}$ are odd integers for every $i, 1 \leq i \leq k$. Hence, since $k$ is an odd integer, $x \cdot y=x_{1} y_{1}+\cdots+x_{k} y_{k}$ is an odd integer, and thus $x \cdot y=x_{1} y_{1}+\cdots+x_{k} y_{k} \neq 0$ in $Z_{n}$, since $n$ is even. Thus $U D(R)$ is completely disconnected.

Theorem 3.4. Let $n \geq 4$ be an even integer, and let $R=Z_{n} \times Z_{n}$. Then the vertex $(n / 2, n / 2)$ in $Z D(R)$ is adjacent to every vertex in $U D(R)$.

Proof. It is clear that $\left(\frac{n}{2}, \frac{n}{2}\right)$ is a vertex of $Z D(R)$. Let $u \in U\left(Z_{n}\right)$. Since $n$ is even, $u$ is an odd integer. Thus $u-1=2 m$ for some integer $m$. Hence $\frac{n}{2}(u-1)=$ $\frac{n}{2}(2 m)=m n=0 \in Z_{n}$. Thus $\frac{n}{2} u=\frac{n}{2}$ in $Z_{n}$. Now let $(a, b) \in U(R)$. Then $a, b \in U\left(Z_{n}\right)$ are odd integers. Hence $(a, b)\left(\frac{n}{2}, \frac{n}{2}\right)=\frac{n}{2}+\frac{n}{2}=n=0 \in Z_{n}$. Thus the vertex $(n / 2, n / 2)$ in $Z D(R)$ is adjacent to every vertex in $U D(R)$.

Example 3.5. Let $A=Z_{8}$ and $R=A \times A$. Then $U D(R)$ is the disjoint union of two $K_{4,4}$ by Theorem 3.2(1). The following is the graph of $U D(R)$. 

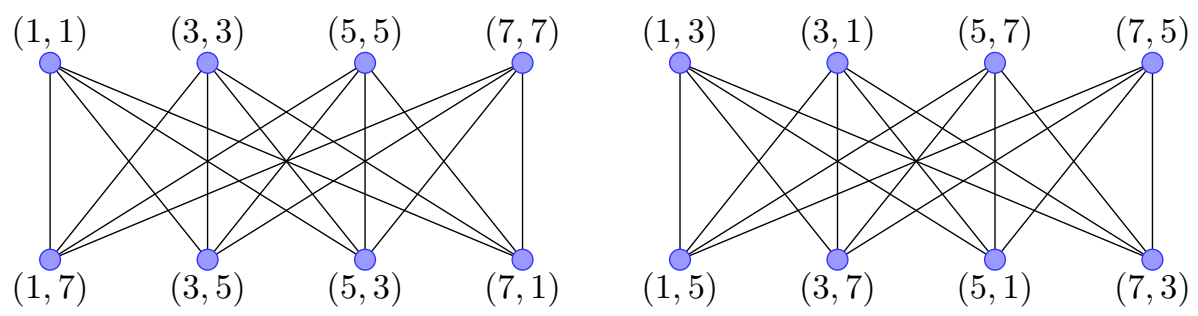

Example 3.6. Let $A=Z_{10}$ and $R=A \times A$. Then $U D(R)$ is the disjoint union of two $K_{4}$ and one $K_{4,4}$ by Theorem 3.2(3, Case I). The following is the graph of $U D(R)$.
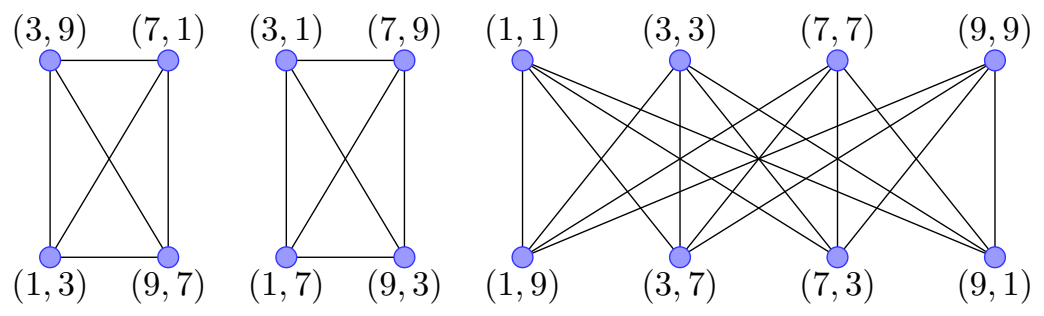

\section{Subgraphs of the zero-divisor dot product graph of $Z_{n} \times Z_{n}$}

For an integer $n \geq 2$, let $R_{1}=\left\{\left(u_{1}, z_{1}\right) \mid u_{1} \in U\left(Z_{n}\right)\right.$ and $\left.z_{1} \in Z\left(Z_{n}\right)\right\}$ and $R_{2}=\left\{\left(z_{2}, u_{2}\right) \mid u_{2} \in U\left(Z_{n}\right)\right.$ and $\left.z_{2} \in Z\left(Z_{n}\right)\right\}$. It is clear that $R_{1} \subset Z\left(Z_{n} \times Z_{n}\right)$ and $R_{2} \subset Z\left(Z_{n} \times Z_{n}\right)$. In this section, we study the induced subgraph $Z D\left(R_{1} \cup R_{2}\right)$ of $Z D\left(Z_{n} \times Z_{n}\right)$ with vertices $R_{1} \cup R_{2}$.

Theorem 4.1. Let $n \geq 2, R=Z_{n} \times Z_{n}$, and $\phi(n)=m$. Then

(1) If $n$ is prime, then $Z D\left(R_{1} \cup R_{2}\right)=Z D\left(Z_{n} \times Z_{n}\right)=\Gamma(R)=K_{n-1, n-1}$.

(2) If $n$ is not prime, then $Z D\left(R_{1} \cup R_{2}\right)$ is the disjoint union of of $(n-m)$ $K_{m, m}$ 's.

Proof. (1) Suppose that $n$ is prime. Then it is clear that $R_{1} \cup R_{2}=Z\left(Z_{n} \times Z_{n}\right)$. If $n=2$, then it is trivial to see that $Z D\left(R_{1} \cup R_{2}\right)=Z D\left(Z_{n} \times Z_{n}\right)=\Gamma(R)=K_{1,1}$. If $n \geq 3$, then the claim is clear by Corollary 2.3(1).

(2) Let $A=Z_{n}$. Suppose that $n$ is not prime. It is clear that every two vertices in $R_{i}$ are not adjacent for every $i \in\{1,2\}$. Let $v_{1} \in R_{1}$ and $v_{2} \in R_{2}$. Then $v_{1}=u(1, a) \in R_{1}$ and $v_{2}=v(b, 1) \in R_{2}$ for some $u, v \in U(A)$ and some $a, b \in Z(A)$. Then $v_{1}$ is adjacent to $v_{2}$ if and only if $v_{1} \cdot v_{2}=u v b+u v a=0$ in $A$ if and only if $b=-a$ in $A$. Hence for each $a \in Z(A)$, let $X_{a}=\{u(1, a) \mid u \in U(A)\}$ and $Y_{a}=\{u(-a, 1) \mid u \in U(A)\}$. It is clear that $\left|X_{a}\right|=\left|Y_{a}\right|=m$. For each 
$a \in Z(A), X_{a} \cap Y_{a}=\emptyset$, every two distinct vertices in $X_{a}$ are not adjacent, and every two distinct vertices in $Y_{a}$ are not adjacent. By construction, it is clear that every vertex in $X_{a}$ is adjacent to every vertex in $Y_{a}$. Thus the vertices in $X_{a} \cup Y_{a}$ form the graph $K_{m, m}$ that is a complete bi-partite subgraph of $Z D(R)$. Since $\left|R_{1}\right|=\left|R_{2}\right|=m(n-m)$ and $R_{1} \cap R_{2}=\emptyset$, we have $\left|R_{1} \cup R_{2}\right|=2 m(n-m)$. Thus $Z D\left(R_{1} \cup R_{2}\right)$ is the disjoint union of of $(n-m) K_{m, m}$ 's.

\section{Equivalence dot product graph}

Let $A=Z_{n}$ and $R=A \times A$. Define a relation $\sim$ on $U(R)$ such that $x \sim y$, where $x, y \in U(R)$, if $x=(c, c) y$ for some $(c, c) \in U(R)$. It is clear that $\sim$ is an equivalence relation on $U(R)$. If $S$ is an equivalence class of $U(R)$, then there is an $a \in U(A)$ such that $S=\overline{(1, a)}=\left\{u(1, a) \mid u \in U\left(Z_{n}\right)\right\}$. Let $E(U(R))$ be the set of all distinct equivalence classes of $U(R)$. We define the equivalence unit dot product graph of $U(R)$ to be the (undirected) graph $E U D(R)$ with vertices $E(U(R))$, and two distinct vertices $X$ and $Y$ are adjacent if and only if $a \cdot b=0 \in A$ for every $a \in X$ and every $b \in Y$ (where $a \cdot b$ denote the normal dot product of $a$ and $b$ ). We have the following results.

Theorem 5.1. Let $n \geq 1, m=2^{n}-1$ and $R=G F\left(2^{n}\right) \times G F\left(2^{n}\right)$. Then $E U D(R)$ is the disjoint union of one $K_{1}$ and $\left(2^{(n-1)}-1\right) K_{1,1}$ 's.

Proof. Let $A=G F\left(2^{n}\right)$. For each $a \in U(A)$, let $X_{a}$ and $Y_{a}$ be as in the proof of Theorem 2.1. Then $X_{a}, Y_{a} \in E(U(R))$. Since $|X|=m$ for each $X \in E(U(R))$, we conclude that each $K_{m}$ of $U D(R)$ is a $K_{1}$ of $E U D(R)$ and each $K_{m, m}$ of $U D(R)$ is a $K_{1,1}$ of $E U D(R)$. Hence the claim follows by the proof of Theorem 2.1.

Theorem 5.2. Let $p \geq 3$ be a positive prime integer, $n \geq 1, m=p^{n}-1$, and let $R=G F\left(p^{n}\right) \times G F\left(p^{n}\right)$. Then

(1) If $4 \nmid m$, then $\operatorname{EUD}(R)$ is the disjoint union of $m / 2 K_{1,1}$ 's.

(2) If $4 \mid m$, then $\operatorname{EUD}(R)$ is the disjoint union of two $K_{1}$ 's and $(m-2) / 2$ $K_{1,1}$ 's.

Proof. Let $A=G F\left(p^{n}\right)$. For each $a \in U(A)$, let $X_{a}$ and $Y_{a}$ be as in the proof of Theorem 2.2. Then $X_{a}, Y_{a} \in E(U(R))$. Since $|X|=m$ for each $X \in E(U(R))$, we conclude that each $K_{m}$ of $U D(R)$ is a $K_{1}$ of $E U D(R)$ and each $K_{m, m}$ of $U D(R)$ is a $K_{1,1}$ of $E U D(R)$. Hence the claim follows by the proof of Theorem 2.2. 
Theorem 5.3. Let $n \geq 3$ be an integer, $R=Z_{n} \times Z_{n}$ and $\phi(n)=m$. Write $n=p_{1}^{k_{1}} p_{2}^{k_{2}} \cdots p_{r}^{k_{r}}$, where the $p_{i}$ 's are distinct prime positive integers, $1 \leq i \leq r$. Then

(1) If $4 \mid n$, then $\operatorname{EUD}(R)$ is the disjoint union of $m / 2 K_{1,1}$ 's.

(2) If $4 \nmid n$ and $4 \nmid\left(p_{i}-1\right)$ for at least one of the $p_{i}$ 's in the prime factorization of $n$, then $\operatorname{EUD}(R)$ is the disjoint union of $m / 2 K_{1,1}$ 's.

(3) If $4 \nmid n$ and $4 \mid\left(p_{i}-1\right)$ for all the odd $p_{i}$ 's in the prime factorization of $n$, then we consider two cases:

Case I. If $n$ is even, then $\operatorname{EUD}(R)$ is the disjoint union of $(m / 2)-2^{r-2}$ $K_{1,1}$ 's and $2^{r-1} K_{1}$ 's.

Case II. If $n$ is odd, then $\operatorname{EUD}(R)$ is the disjoint union of $(m / 2)-2^{r-1}$ $K_{1,1}$ 's and $2^{r} K_{1}$ 's.

Proof. Let $A=Z_{n}$. For each $a \in U(A)$, let $X_{a}$ and $Y_{a}$ be as in the proof of Theorem 3.2. Then $X_{a}, Y_{a} \in E(U(R))$. Since $|X|=m$ for each $X \in E(U(R))$, we conclude that each $K_{m}$ of $U D(R)$ is a $K_{1}$ of $E U D(R)$ and each $K_{m, m}$ of $U D(R)$ is a $K_{1,1}$ of $E U D(R)$. Hence the claim follows by the proof of Theorem 3.2.

Let $R_{1}=\left\{\left(u_{1}, z_{1}\right) \mid u_{1} \in U\left(Z_{n}\right)\right.$ and $\left.z_{1} \in Z\left(Z_{n}\right)\right\}$ and $R_{2}=\left\{\left(z_{2}, u_{2}\right) \mid u_{2} \in\right.$ $U\left(Z_{n}\right)$ and $\left.z_{2} \in Z\left(Z_{n}\right)\right\}$, see Section 4. We define a relation $\sim$ on $R_{1} \cup R_{2}$ such that $x \sim y$, where $x, y \in R_{1} \cup R_{2}$, if $x=(c, c) y$ for some $(c, c) \in U\left(Z_{n} \times Z_{n}\right)$. It is clear that $\sim$ is an equivalence relation on $R_{1} \cup R_{2}$. By construction of $R_{1}$ and $R_{2}$, it is clear that if $x \sim y$ for some $x, y \in R_{1} \cup R_{2}$, then $x, y \in R_{1}$ or $x, y \in R_{2}$. Hence if $S$ is an equivalence class of $R_{1} \cup R_{2}$, then there is an $a \in Z\left(Z_{n}\right)$ such that either $S=\left(\overline{(1, a)}=\left\{u(1, a) \mid u \in U\left(Z_{n}\right)\right\}\right.$ or $S=\overline{(a, 1)}=\left\{u(a, 1) \mid u \in U\left(Z_{n}\right)\right\}$. Let $E\left(R_{1} \cup R_{2}\right)$ be the set of all distinct equivalence classes of $R_{1} \cup R_{2}$. We define the equivalence zero-divisor dot product graph $R_{1} \cup R_{2}$ to be the (undirected) graph $E Z D\left(R_{1} \cup R_{2}\right)$ with vertices $E\left(R_{1} \cup R_{2}\right)$, and two distinct vertices $X$ and $Y$ are adjacent if and only if $a \cdot b=0 \in A$ for every $a \in X$ and every $b \in Y$ (where $a \cdot b$ denote the normal dot product of $a$ and $b$ ). We have the following result.

Theorem 5.4. Let $n \geq 2, R=Z_{n} \times Z_{n}$, and $\phi(n)=m$. Then

(1) If $n$ is prime, then $E Z D\left(R_{1} \cup R_{2}\right)=K_{1,1}$.

(2) If $n$ is not prime, then $E Z D\left(R_{1} \cup R_{2}\right)$ is the disjoint union of of $(n-m)$ $K_{1,1}$ 's.

Proof. (1) If $n$ is prime, then $E=\{\overline{(1,0)}, \overline{(0,1)}\}$. Thus $E Z D\left(R_{1} \cup R_{2}\right)=K_{1,1}$.

(2) Suppose that $n$ is not prime, and let $A=Z_{n}$. For each $a \in Z(A)$, let $X_{a}$ and $Y_{a}$ be as in the proof of Theorem 4.1. Then $X_{a}, Y_{a} \in E\left(R_{1} \cup R_{2}\right)$. Since $|X|=m$ 
for each $X \in E\left(R_{1} \cup R_{2}\right)$, we conclude that each $K_{m, m}$ of $Z D\left(R_{1} \cup R_{2}\right)$ is a $K_{1,1}$ of $\operatorname{EZD}\left(R_{1} \cup R_{2}\right)$. Hence the claim follows by the proof of Theorem 4.1.

Remark 5.5. (1) Let $A=Z_{n}$ and $R=Z_{n} \times Z_{n}$. Since for each $X \in E(U(R))$ there exists an $a \in U(A)$ such that $X=\overline{(1, a)}=\{u(1, a) \mid u \in U(A)\}$, note that we can recover the graph $U D(R)$ from the graph $E U D(R)$. However, drawing $E U D(R)$ is much simpler than drawing $U D(R)$.

(2) Since for each $X \in E\left(R_{1} \cup R_{2}\right)$ there exists an $a \in Z\left(Z_{n}\right)$ such that either $X=\overline{(1, a)}=\left\{u(1, a) \mid u \in U\left(Z_{n}\right)\right\}$ or $X=\overline{(a, 1)}=\{u(a, 1) \mid u \in$ $\left.U\left(Z_{n}\right)\right\}$, note that we can recover the graph $Z D\left(R_{1} \cup R_{2}\right)$ from the graph $E Z D\left(R_{1} \cup R_{2}\right)$. However, drawing $E Z D\left(R_{1} \cup R_{2}\right)$ is much simpler than drawing $Z D\left(R_{1} \cup R_{2}\right)$.

Example 5.6. Let $A=Z_{20}$ and $R=A \times A$. Then $\operatorname{EUD}(R)$ is the disjoint union of $4 K_{1,1}$ by Theorem $5.3(1)$, and thus $U D(R)$ is the disjoint union of $4 K_{8,8}$. The following is the graph of $E U D(R)$.
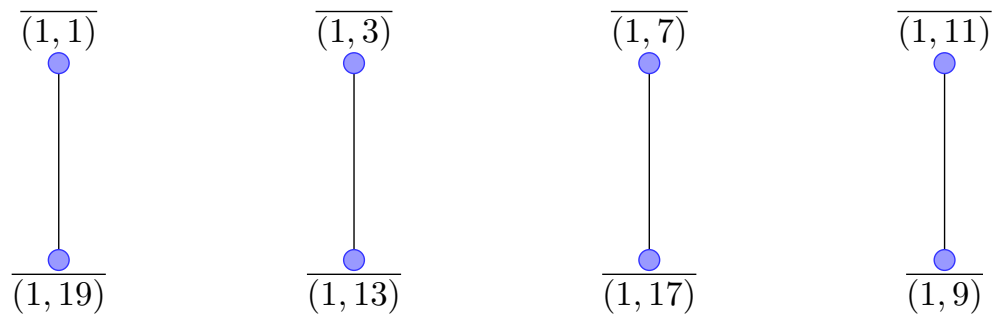

Example 5.7. Let $A=Z_{34}$ and $R=A \times A$. Then $\operatorname{EUD}(R)$ is the disjoint union of $7 K_{1,1}$ 's and $2 K_{1}$ 's by Theorem $5.3(3$, Case I), and thus $U D(R)$ is the disjoint union of $7 K_{16,16}$ and $2 K_{8}$. The following is the graph of $E U D(R)$.

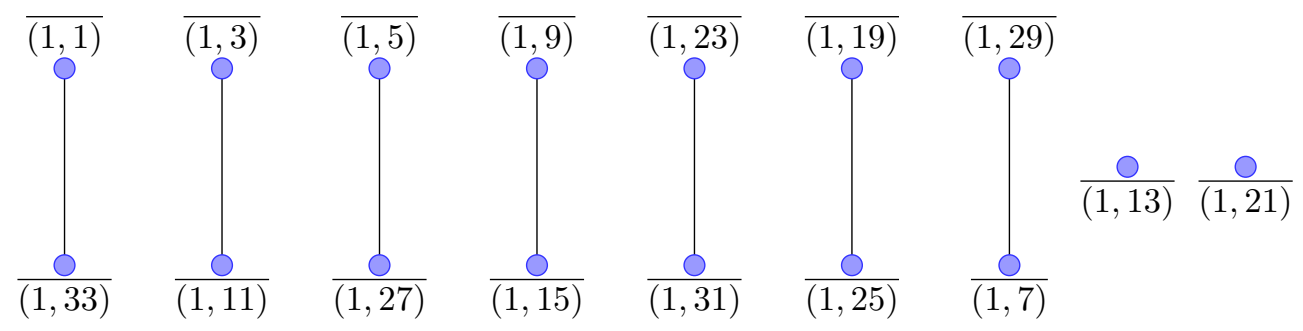

Acknowledgement. We are so grateful to the referee for his/her great effort in proof reading the manuscript. 


\section{References}

[1] D. D. Anderson and M. Naseer, Beck's coloring of a commutative ring, J. Algebra, 159 (1993), 500-514.

[2] D. F. Anderson and A. Badawi, On the zero-divisor graph of a ring, Comm. Algebra, 36 (2008), 3073-3092.

[3] D. F. Anderson and A. Badawi, The total graph of a commutative ring, J. Algebra, 320 (2008), 2706-2719.

[4] D. F. Anderson and A. Badawi, On the total graph of a commutative ring without the zero element, J. Algebra Appl., 11(4) (2012), 1250074 (18 pp).

[5] D. F. Anderson and A. Badawi, The generalized total graph of a commutative ring, J. Algebra Appl., 12(5) (2013), 1250212 (18 pp).

[6] D. F. Anderson and J. D. LaGrange, The semilattice of annihilator classes in a reduced commutative ring, Comm. Algebra, 43 (2015), 29-42.

[7] D. F. Anderson and J. D. LaGrange, Some remarks on the compressed zerodivisor graph, J. Algebra, 447 (2016), 297-321.

[8] D. F. Anderson and E. F. Lewis, A general theory of zero-divisor graphs over a commutative ring, Int. Electron. J. Algebra, 20 (2016), 111-135.

[9] D. F. Anderson and P. S. Livingston, The zero-divisor graph of a commutative ring, J. Algebra, 217 (1999), 434-447.

[10] D. F. Anderson and S. B. Mulay, On the diameter and girth of a zero-divisor graph, J. Pure Appl. Algebra, 210 (2007), 543-550.

[11] D. F. Anderson and D. Weber, The zero-divisor graph of a commutative ring without identity, Int. Electron. J. Algebra, 23 (2018), 176-202.

[12] S. Akbari, H. R. Maimani and S. Yassemi, When a zero-divisor graph is planar or a complete r-partite graph, J. Algebra, 270 (2003), 169-180.

[13] S. Akbari, D. Kiani, F. Mohammadi and S. Moradi, The total graph and regular graph of a commutative ring, J. Pure Appl. Algebra, 213 (2009), 2224-2228.

[14] A. Badawi, On the annihilator graph of a commutative ring, Comm. Algebra, 42 (2014), 108-121.

[15] A. Badawi, On the dot product graph of a commutative ring, Comm. Algebra, 43 (2015), 43-50.

[16] I. Beck, Coloring of commutative rings, J. Algebra, 116 (1988), 208-226.

[17] C. F. Kimball and J. D. LaGrange, The idempotent-divisor graphs of a commutative ring, Comm. Algebra, 46 (2018), 3899-3912.

[18] J. D. LaGrange, The $x$-divisor pseudographs of a commutative groupoid, Int. Electron. J. Algebra, 22 (2017), 62-77. 
[19] W. J. LeVeque, Fundamentals of Number Theory, Addison-Wesley Publishing Company, Reading, Massachusetts, 1977.

[20] S. B. Mulay, Cycles and symmetries of zero-divisors, Comm. Algebra, 30 (2002), 3533-3558.

[21] R. Nikandish, M. J. Nikmehr and M. Bakhtyiari, Coloring of the annihilator graph of a commutative ring, J. Algebra Appl., 15(7) (2016), 1650124 (13 pp).

[22] Z. Pucanović and Z. Petrović, On the radius and the relation between the total graph of a commutative ring and its extensions, Publ. Inst. Math. (Beograd) (N.S.), 89 (2011), 1-9.

[23] P. K. Sharma and S. M. Bhatwadekar, A note on graphical representation of rings, J. Algebra, 176 (1995), 124-127.

[24] M. Sivagami and T. Tamizh Chelvam, On the trace graph of matrices, Acta Math. Hungar., 158 (2019), 235-250.

[25] S. Spiroff and C. Wickham, A zero divisor graph determined by equivalence classes of zero divisors, Comm. Algebra, 39 (2011), 2338-2348.

[26] T. Tamizh Chelvam and T. Asir, On the genus of the total graph of a commutative ring, Comm. Algebra, 41 (2013), 142-153.

Mohammad Abdulla and Ayman Badawi (Corresponding Author)

Department of Mathematics and Statistics

The American University of Sharjah

P.O. Box 26666, Sharjah, United Arab Emirates

emails: Mohammadabdulla85@hotmail.com (M. Abdulla)

abadawi@aus.edu (A. Badawi) 